\title{
ZUR GEGENWÄRTIGEN SITUATION DER ÖSTERREICHISCHEN THEATER
}

\author{
Dr. HANS BRUNMAYR
}

Ausländer, die entsprechende Kontakte zur Wiener Bevölkerung gewonnen haben, wundern sich darüber, dass eine Direktionskrise in der Wiener Staatsoper die Gemüter viel mehr erregt als etwa der Rücktritt eines Ministers oder das Ergebnis einer Parlamentswahl. Der Ausländer staunt wohl auch darüber, dass bei dem Begräbnis eines prominenten Mitgliedes des Wiener Burgtheaters ein eigenes Zeremoniell angewendet wird. Während sonst die Aufbahrung der Toten auf den Friedhöfen vorgeschrieben ist, wird der Sarg mit dem toten Mimen im Burgtheater aufgstellt und dann durch ein von Tausenden gebildetes Spalier einmal rund um das Theater gefahren. Eine änhliche ehrende Sonderbehandlung, nämlich der Kondukt über die prunkvolle Ringstrasse, wird, sonst nur dem während seiner Amtszeit verstorbenen Staatsoberhaupt zuteil. Ich habe das Estraunen über die Reaktion der Bevölkerung auf die Direktionskrise und auf den Tod eines berühmten Schauspielers einem Ausländer zugeschrieben. Die meisten Wiener wundern sich über diese Reaktion nicht, sie erscheint ihnen mehr oder weniger selbstverständlich. Mit diesem anekdotischen Beginn meines-im Folgenden viel sachlicheren-Referats wollte ich ihnen zu erkennen geben, dass dem Theater im österreichischen Alltag ganz offensichtlich eine besondere Position und Funktion zugebilligt wird und dass man das Ansehen und das Sozialprestige eines grossen Schauspielers nur als ausserordentlich bezeichnen kann. Freilich meine beiden Beispiele bezogen sich auf die Wiener Staatsoper und auf das Burgtheater, also auf jene Bühnen, die bis 1918 Hoftheater waren und seither vom Staat erhalten werden. Da die Republik Österreich ein Bundesstaat ist, werden diese Bühnen als Bundestheater bezeichnet. Diese nehmen - was ihr Ansehen und die Anteilnahme des Publikums an ihnen betrifft - innerhalb der österreichischen Theater eine eindeutige Sonderstellung ein; eine Sonderstellung, die keineswegs eine kritische Haltung des Publikums aus- 
schliesst. Dieses möchte ja die Sonderstellung immer wieder durch besondere Leistungen bestätigt sehen. Von den österreichischen Bundestheatern heisst es, dass sie den grössten Theaterkonzern der Welt darstellen. Auch darüber freuen sich die meisten kulturell interessierten Österreicher. Sie sind stolz, dass ihr nach 1918 kleingewordenes Vaterland die künstlerische Repräsentanz einer Grossmacht erfolgreich aufrecht erhalten und fortführen konnte.

Die Freude wird allerdings getrübt, wenn der österreichische Steuerzahler erfährt, dass das Budget des Staates für 1970 Ausgaben der Bundestheater von mehr als 530 Millionen Schilling, das sind mehr als ca 250 Mill T Lira vorsieht. Die Einnahmen werden mit rund 160 Millionen Schilling, das sind etwa 80 Mill T Lira präliminiert. Es ergibt sich also ein Verlust von über 370 Millionen Schilling, das sind über 185 Mill T Lira. Das bedeutet, dass jeder der rund 300 Spieltage pro Jahr - die Bundestheater spielen auf vier Bühnen - für den Staat, mit einem Verlust von rund 1, 1/4 Millionen Schilling, das sind über 600.000 TL verbunden ist. Es ist begreiflih, dass man nach Wegen sucht, die zu einer Verringerung dieses Defizits führen könnten, und dass man sich fragt, ob der enorme Betrag für die Budestheater nicht effektiver als bisher angewendet werden könnte. Es ist daher vor kurzem eine Umorganisation der Bundestheater in Angriff genommen worden. Zweifellos wird dabei die künstlerische Entscheidungsfreiheit der Direktoren der einzelnen Bühnen und auch ihr finanzielles Pouvoire aufrecht erhalten werden. Das Ziel der Reform wird vor allem eine Vereinfachung und Rationalisierung des Verwaltungsapparates sein. Auch möchte man die Ausgaben für jene Künstler reduzieren, deren Einssatzmöglichkeit - etwa aus Altersgründen - nur mehr gering ist.Durch ein Gewohnheitsrecht wird dem an den österreichischen Bundestheatern tätigen Künstlern nach zehnjähriger ununterbrochener $\mathrm{Zu}$ gehörigkeit und jeweils mindestens 8 monatiger Anwesenheit die Pensionsberechtigung zugebilligt. Um aber die Bemessungsgrundlage für die Pensionen hoch zu halten, werden meistens die Gagen nicht verringert, wenn die künstlerische Leistungfähigkeit abnimmt. Reformbestrebungen, die auf finanzielle Einbussen für Künstler hinauslaufen, werden nicht nur in Künstlerkreisen auf starken Widerstand stossen, sie werden auch in Kreisen des Theaterpublikums, das in Wien seinen Bühnenlieblingen über Jahrzehnte die Treue hält, unpopulär sein.

\section{Staatsoper und Volksoper}

Das in der Welt bekannteste österreichische Bundestheater ist die Wiener Staatsoper, eine der berühmtesten Pflegestätten des $\mathrm{Mu}$ - 
siktheaters. (s. Bild. 1) Sie hat in der vergangenen Spielzeit das hundertjährige Jubiläum ihres reprasentativen Heimes, des Operngebäudes an der Wiener Prunkstrasse, dem Ring, gefeiert. Dieses Haus, hat wähZweiten Weltkrieges bei einem amerikanischen Luftangriff schwerste rend des. Schaden erlitten, Es wurde -unter Bedachtnahme auf seine historische Gestalt-wiedererrichtet und 1955 neueröffnet. Vorher hatte die Staatsoper ein Jahrzehnt in einem Ausweichquartier gespielt. Dort wurde besondere Liebe und Sorgfalt an die Aufführungen von Mozart Opern gewendet. Es standen gerade für diese Aufgabe pradestinierte, hervorragende Sänger zur Verfügung, die sich zu einem unvergleichlichen Ensemble vereinigten. Man sprach sehr bald von dem Wiener Mozartstil, der als Höhepunkt der stilistischer Authentizitat angesehen wurde. Durch Gastspiele im Ausland, durch Aufführungen der Salzburger Festspiele und durch Schallplatten wurde dieser Wiener Mozartstil in der ganzen Welt bekannt. Es ist unverkennbar, dass er die Mozart-Interpretation in aller Welt nachhaltig beeinflusst hat. Sehr bald nach der Ưbersiedlung in das erneuerte alte Haus am Ring zeigte es sich aber - vor allem bei Verdi-und Wagner-Aufführungen - dass ein Opernhaus von Spitzenrang nicht mehr als Ensembletheater, zumindest nicht mehr als reines Ensembletheater, geführt werden könne, dass es sich mehr oder weniger dem Startheater nähern müsse. Der Flugverkehr hat es nun einmal möglich gemacht, dass Stars im Laufe einer Saison in den bekanntesten Opernhäusern und bei den angesehensten Festspielen vor das Publikum treten. Und ein Opernhaus, das etwa glauben sollte, auf derartige Stars verzichten zu können, würde sehr bald nicht mehr zur Weltspitze gehören. Der Glücksfall der Wiener Mozartaufführungen, die noch einmal alle Vorzüge des Ensembletheaters in höchster Vollkommenheit manifestierten, konnte über den möchtigen Trend zum Startheater nicht hinwegtauschen*. Herbert von Karajan, hat während der glanzvollen Aera seiner Direktion an der Wiener Staatsoper die Konsequenz aus diesen Trend gezogen und den Schritt in das Startheater entschlossen getan. Selbstverständlich hat das Startheater eine Nivellierung der Opernspielpläne zur Folge. Allerdings war ja auch schon vor der Entwicklung zu Startheater die Differenzierung in den Spielplänen der Operntheater gering. Gerade die Wiener Staatsoper beschränkte sich seit jeher auf ein Standartrepertoire, weil sie sich wegen des konservativen Geschmacks der Mehrzehl ihrer Besucher der Pflege der modernen Oper nur selten gewidmet hat-

* Man darf ja auch nicht übersehen, dass die Träger der Hauptrollen in diesem Ensemble durchwegs zu international anerkannten Stars geworden waren. 
te. Sehr freulich ist es, dass sich der zur Wiedereröffnung 1955 neu eintudierte "Wozzek" des österreichischen Komponisten Alban Berg (1885-1935) auf dem Spielplan erhalten hat und sich zunehmenden Publikumsinteresses erfreut. Die Aufführung dieses Werkes ist allerdings als derzeitige Spitzenleistung der Wiener Staatsoper anzusehen. Seither hat man auch "Lulu" von Alban Berg ebenfalls in einer exemplarischen Aufführung herausgebracht. Man dàrf hoffen, dass auch dieses Werk zum fixen Repertoire gehören wird.

Das von Jahr zu Jahr trotz guten Besuchs ständig steigende Defizit der Bundestheater hat an der Wiener Staatsoper zu einer bedauerlichen Drosselung der Neuinszenierungen geführt. Die Vermehrung des Defizits wird verständlich, wenn man weiss, wie gross etwa bei der Wiener Staatsoper die Anzahl der Personen ist, denen Gagen-und Lohnerhöhungen - im Gefolge einer allgemeinen Steigerung der Lebenshaltungskosten - zugute kommen. Die Wiener Staatsoper verfügt über mehr als 100 Gesangsolisten zu denen noch etwa 25 regelmässig gastierende Künstler kommen. Das Orchester besteht aus 152 Musikern, ausserdem sind 23 Bühnenmusiker engagiert. Dem Ballett gehören 85 Tänzerinnen und Tänzer an, der Chor hat über 100 Mitglieder. Zusammen mit den Angestellten der Direktion, mit Dirigenten und Korrepetitoren, mit Regisseuren und deren Assistenten, mit Bühnen-und Kostümbildnern und mit der Komparserie ergibt sich die Zahl von ca. 1200 Personen. Zählt man dazu noch das technische Personal von ca. 560, so ergibt sich für die Wiener Staatsoper ein Stand von ca. 1760 Beschäftigten. Besondere personelle und finanzielle Probleme werden für die Wiener Staatsoper und für alle Theater in Österreich durch eine schrittweise Verkürzung der Arbeitszeit auf 40 Stunden pro Woche entstehen.

Das zweite staatliche Musiktheater, die Volksoper hält im Wesentlichen noch am Ensemble fest. Allerdings spielen auch hier in einzelnen Produktionen neben Solisten aus dem Stammpersonal ganze Ensemblegruppen amerikanischer Künstler (z. B. West-Side-story). Seit ihrer Wiedereröffnung nach dem Zweiten Weltkrieg hatte sich diese Bühne vorwiegend der Operette gewidmet. Es wurde hier ein beispielhafter Stil für die Aufführung klassischer Wiener Operetten (von Johann Strauss, Millöcker und Suppé) erarbeitet. Hier wurden auch die ersten Musicals in Wien aufgeführt. Daneben hatte aber auch die Spieloper ihre Heimstätte. Erst die derzeitige Direktion hat die Position der Oper im Spielplan wesentlich verstärkt. Es werden vor allem selten gespielte, fast vergessene Werke dieser Gattung, in das Repertoire aufgenommen. 


\section{Burgtheater und Akademietheater}

Das berühmteste österreichische Sprechtheater lässt schon durch den Namen "Burgtheater" seine einstige Funktion als Bühne des kai-serlichen Hofes erkennen (s. Bild. 2). Es wurde von Kaiser Joseph II. im Jahre 1776 gegründet und ist seit 1888 in dem grossen Prunkbau an der Ringstrasse untergebracht. Wie die Staatsoper wurde auch das Burgtheater im Zweiten Weltkrieg schwer beschädigt und 1955 wieder eröffnet. Dieses Theater trägt den Ruhm einer grossen Tradition, trägt aber auch die Lasten einer ruhmreichen Vergangenheit. Zu diesen Lasten gehört auch eine sehr typische Einstellung des Publikums zu dieser Bühne. Aus dem Wissen um die grosse Vergangenheit dieses Theaters hat sich das Stammpublikum daran gewöhnt besondere Ansprüche zu stellen. Es hat sich eine utopische Vorstellung entwickelt, an deren Realisierung in der Vergangenheit dieses Publikums glaubt. Für die Gegenwart wird aus dieser Vorstellung ein Wunschbild abgeleitet. Aus der Diskrepanz zwischen ihm und der Realität des Bühnenalltags entsteht etwas wie eine leicht enttäuschte, ein wenig unglückliche liebe. Das Stammpublikum wäre aber nicht bereit, einem gelegentlichen Besucher das Recht einzuräumen, von einer Aufführung im Burgtheater enttäuscht $\mathrm{zu}$ sein.

Traditionellerweise pflegt das Burgtheater die grosse dramatische Weltliteratur, die Klassiker. Der Inhalt dieses Begriffs darf allerdings nicht zu eng gefasst werden. Traditionellerweise weicht der Spielplan des Burgtheaters dem Modernem in einigermassen extremer oder experimenteller Ausprägung aus. Und es tut gut daran. Die Atmosphäre des traditionsreichen Hauses aber auch der durch die klassischen Werke geprägte Darstellungsstil der Schauspieler stünden einem Erfolg derartiger moderner Werke entgegen.

Es soll nicht verschwiegen werden, dass dem Burgtheater oftmals der Vorwurf gemacht wird, eine museale Existenz zu führen und aufrecht erhalten zu wollen. Tatsächlich kann man die Frage nicht einfach abtun, ob ein grosses Klassikertheater in der Gegenwart noch eine lebensvolle Funktion zu erfüllen habe. Es ist nicht zu verkennen, dass die Faszination des Burgtheaters auf die Jugend in den letzten Jahren nachgelassen hat. Aber auch bei den älteren Theaterbesuchern fanden die während der letzten Spielzeiten gebotenen Neuinszenierungen klassischer Werke eine kühlere Aufnahme.

Erfreulicherweise hat das Burgtheater die grossen Werke des Wiener Volksschauspiels, das früher -als zu vulgär- von Aufführungen 
an der Hofbühne ausgeschlossen war, in seinen klassischen Spielplan integriert . Werke von Ferdinand Raimund und Johann Nestroy wurden in vorbildlichen Aufführungen dargeboten. Das Burgheater besitzt in seinem grossen Ensemble eine ganze Reihe hervorragende Schauspielerpersönlichkeiten. Das Interesse am Schauspieler und an seiner Leistung ist in Wien seit altersher viel reger als das an dem Autor und seinem Werk. Die grossen Schauspielern des Burgtheaters sind freilich meistens Künstler in reiferen Jahren. Unter den jungen sind mitreissende Persönlichkeiten selten zu finden. Auch das mag ein Grund dafür sein, dass - wie ich schon sagte - die Faszination des Burgheaters auf die Jugend schwächer wurde.

Das Burgtheater besitzt im Akademietheater eine Filialbühne von intimer Atmosphäre. Hier wird das Kammerspiel, die Komödie und moderne Dramatik-gelegentlich auch absurdes Theater-gepflegt. Es war von dem Stammpublikum des Burgtheaters die Rede. Diesem wird durch ein Abonnementssystem die Möglichkeit zu einem allerdings nur um $10 \%$ verbiligten Besuch von 7 Vorstellungen in der Saison geboten. Auch im Akademietheater umfasst das Abonnement sieben Vorstellungen. Die beiden staatlichen Opernbühnen bieten ihren Abonnenten je 6 Vorstellungen, ebenfalls mit einer Verbilligung von $10 \%$. An Jedem österreichischen Bundestheater werden derzeit -je nach Vorstellung únd Besetzung - vier verschiedene Preistarife angewendet. Der höchste Tarif beträgt für jede Platzkategorie jeweils das Doppelte des niedrigsten Tarifs. Als Grundlage für die Berechnung der Abonnementspreise dient an allen Bundestheatern der niedrigste Tarif. Die Abonnenten haben den Vorteil, dass ihnen auch gelegentlich Vorstellungen zugänglich gemacht werden, für die im Einzelverkauf ein höherer Tarif gilt.

\section{Die Wiener Privattheater}

Zur Unterscheidung von den Bundestheatern werden die anderen grösseren Wiener Bühnen, so vor allem die Sprechbühnen "Theater in der Josefstadt", welches auch zwei Filialbühnen bespielt und das "Volkstheater", sowie das "Raimundtheater", eine Operettenbühne, als Privattheater bezeichnet. Allerdings sind diese Bühnen schon längts keine Privattheater im eigentlichen Wortsinn. Es wäre nämlich unmöglich, dass diese Bühnen ihren Betrieb ohne beträchtlichen Subventionen vom Staat und von der Gemeinde Wien aufrecht erhielten. Grund dafür ist dass keine kostendeckende Preise verlangt werden können. Im Theater in der Josefstadt kostete 1938 der teuerste 
Sitz S. 25, - dieser Betrag besass damals eine Kaufkraft von ca. 250 heutigen Schilling. Für die teuerste Karte im Theater in der Josefstadt wird jetzt aber nur etwa die Hälfte dieses Betrages verlangt. Und das auf Grund einer richtigen Überlegung, denn eine Verdoppelung der Preise hätte halbleere Häuser zur Folge. Eine Drosselung der Ausgaben des Theaters ist nicht möglich. Erste Schauspieler könnten, wenn ihnen geringere Gagen geboten würden, nicht an diesem Theater gehalten werden. Die Gagenansprüche sind durch den Vergleich mit Filmund Fernsehenhonoraren und mit den von deutschen und schweizerischen Bühnen gebotenen Summen begreiflicherweise seit 1938 stark gestiegen. Durch die Sozialgesetzgebung wurde die finanzielle Situation des technischen Personals sehr wesentlich gebessert und dadurch sind gleichfalls die Ausgaben des Theaters erhöht worden.

Das Theater in der Josefstadt wurde in den Zwanziger - und frühen Dreissigerjahren von Max Reinhardt, dem grossen Regisseur geleitet. Dass die Vollkommenheit der schauspielerischen Leistung, die vollendete Emsemblewirkung im Mittelpunkt der künstlerischen Bemühungen stand, fand ihren Ausdruck schon in der offiziellen Bezeichnung des Theaters. Diese lautete "Die Schauspieler des Theaters in der Josefstadt unter Führung von Max Reinhardt". Auch heute noch sind die künstlerischen Intentionen des Theaters in erster Linie auf die Pflege eines kultivierten Kammerspieltons, eines Josefstädter Ensemblesstils gerichtet. Deshalb gelangen denn auch in den letzten Jahren einige exemplarische Aufführungen von Werken des bedeutenden österreichischen Dramatikers Arthur Schniztler unter der Regie seines Sohnes Heinrich.

Das Publikum dieses Hauses sucht aber in erster Linie anregende Unterhaltung. Der langjährige Direktor des Hauses ist der Meinung, dass ihm nur das Abonnementssystem die Möglichkeit bietet, literarisch anspruchsvollere Stücke in den Spielplan miteinzubeziehen. Andernfalls wäre der Besuch derartiger Vorstellungen zu schwach, die Aufführungszahl infolgedessen zu niedrig, um die Einstudierung zu rechtfertigen.

- In der einen Filialbühne, den "Kammerspielen" pflegt das Josefstädter Ensemble typisches Boulevardtheater. In dem kleinen Theater im Konzerthaus bepsielt das Theater in der Josefstadt eine zweite Filialbühne und widmet sich dort der modernen Dramatik. Es besteht eine Zusammenarbeit mit dem östereichischen Fernsehen, das daher auch entsprechenden Einfluss auf die Gestaltung des Spielplanes nimmt. 
Dem "Volkstheater", das über ein geräumiges Haus verfügt, wird seit Jahren von der Kritik bescheiningt, dass es den interessantesten Spielplan aller Wiener Sprechbühnen verwirklicht. Tatsächlich stehen hier-ungleich stärker als im Burgtheater oder gar im Theater inder Josefstadt - die Aussage, das Anliegen des Autors und die zeitnahe oder zeitgemässe Problematik im Mittelpunkt. Bei der Spielplangestaltung wird sehr klug dosiert; dem ursprünglichen Anliegen dieser Bühne, dem Wiener Volksstück, wird durch Aufführungen von Stücken Johann Nestroys oder - in seiner zeitnahen Form - durch die Pflege der Dramatik Öden von Horvaths der gebührende Taum im Programm gegeben ${ }^{99} .{ }^{9}$ Aus der klassischen Literatur wird sinnvoll ausgewählt. Das Programm des Volkstheaters berücksichtigt aber besonders Stücke, die sich mit zeitnahen Problemen befassen.

Diese Bühne besitzt ein besonders preisgünstiges Abonnementssystem und zwar ein Abonnement für den Hauptteil des Repertoires und ein sogenanntes Sonderabonnement für drei anspruchsvolle, zu einem Zyklus "Konfrontation" zusammengefasste Stücke. Der niedrige Abonnementpreis ist durch eine Preisstützung durch den österreichischen Gewerkschaftsbund und eine diesem nahestehende Bank ermöglicht.

Das "Raimundtheater" ist die einzige Wiener Bühne, die sich ausschliesslich der einstmals so erfolgreichen und an vielen Wiener Theatern geplegten Operette widmet. Ihr Spielplan basiert auf Werken aus der sogenannten silbernen Aera der Wiener Operette, die etwa durch die erfolgreichen Komponisten Lehar, Oscar Straus, Kalman, Fall, Eysler und Stolz gekennzeichnet ist.

Das Theater an der Wien, eine traditionsreiche Altwiener - Bühne wurde vor einigen Jahren durch die Stadt Wien gründlich renoviert, um zur Zeit der Wienner Festwochen als Festspielhaus für die Eigenproduktionen der Festwochenintendanz zu dienen. Während des grössten Teils der Saison bevorzugte diese Bühne das Musical. Es beherbergaber auch oftmals Gastspieltruppen.

"Die Wiener Kammeroper", die vor allem dem Sängernachwuchs erste Chancen bietet, widmet sich der Aufführung selten gespielter komischer Oper und Singspiele. Es wird kein täglicher Spielbetrieb durchgeführt. Während der Theatersaison steht der Kammeroper ein Theatersaal im Stadtzentrum zur Verfügung, im Hochsommer spielt sie in dem architektonisch reizvollen Schönbrunner Schlosstheater.

Seit Jahren vermag eine Bauernbühne, deren Ensemble sich um mehrere Angehörige der Familie Löwinger gruppiert hat, ein anspruch- 
loses Publikum im Theater und im Fernsehen mit derber Kost zu befriedigen.

Im Sommer produziert sich auf einem mit Planken umzäunten Grundstück am Stadtrand das letzte Wiener Stehgreiftheater. Nach einem kurzen Informationsgespräch treten die im Improvisieren erstaunlich routinierten Darsteller auf und bieten ländliche Lustspiele, Rührstücke oder Tragödien, die beim Publikum wegen der so oft erzielten unfreiwilligen Komik besonders beliebt sind.

\section{Die Kellertheater}

Die Zerstörung vieler Theatergebäude während des Zweiten Weltkrieges wàr der Anlass, behelfsmässig Theaterräume zu adaptieren. Meistens handelte sich um Souterrainlokale, die zu Kaffeehäusern und Restaurants gehören. Manchmal hat man sich aber auch in Kellergewölben von Privathäusern installiert. In Wien waren allerdings schon in den Dreissigerjahren in Kellerräumen von Kaffeehäusern Spielstätten eingerichtet worden, in denen aber durchwegs ein cabaretistisches Genre gepflegt wurde, freilich meistens Cabaret von beträchtlichem literarischen Rang.

Für die Gründung von kleinen Bühnen nach 1945 waren aber nicht nur die Zerstörungen des Krieges und die Not der Zeit massgebend, es gab dafür auch geistige Voraussetzungen. Während der Zeit der nationalsozialistischen Herrschaft war das Theaterpublikum von der Möglichkeit, die ausländische zeitgenössische Dramenliteratur kennenzulernen, fast völlig abgeschnitten. So wie die meisten Werke der modernen bildenden Kunst galten auch viele literarische Werke, die sich moderner Formen bedienten oder angeblich dekadente Themen behandelten, als "entartete Kunst". Sie waren von der öffentlichen Darbietung ebenso ausgeschlossen wie alle Werke jüdischer Autoren. Nach 1945 herrschte also ein sehŕr reger Nachholbedarf. Der Mangel an Konsum-und Luxusgütern, der Ausgaben hiefür weitgehend reduzierte, steigerte die Teilnahme des Publikums an kulturellen Veranstaltungen. Es kam damals zur Gründung vieler Kleinbühnen, sogenannter Kellertheater. Einige von ihnen konnten bedeutende künstlerische Erfolge erzielen. In vielen Fällen erwiesen sich diese kleinen Bühnen für Regisseure und Schauspieler als Sprungbretter zu grossen Karrieren. Die Zahl der Kellertheater schwankt. Es ist nicht allzu schwer mit viel Unternehmungslust und wenig Geld ein derartiges Theater zu gründen. Nicht alle derartigen Untermehmungen erwiesen șich als langlebig. Zur Zeit spielen in der österreichischen Hauptstadt etwa zehn 
solcher Kellertheater. Einige widmen sich sinnvollerweise Werken der Avantgarde, andere spielen vorwiegend konservatives Theater auf konventionelle Weise und gehen so an den künstlerischen Möglichkeiten und Aufgaben der Kellertheater vorbei. In einem der Wiener Kellertheater entwickelte das Ensemble "Die Komödianten" einen typischen, sich der Pantomine nähernden Darstellungsstil.

Die Spielplangestaltung der Kellertheater wird dadurch erschwert, dass die Bühnenverlage Stücke, für die sie die Annahme durch eine grosse Bühne und daher beträchtliche Tantiemeneingänge erhoffen, nicht an Kleinbühnen vergeben. Die Kellertheater verfügen nicht über fix angagierte Ensembles die Schauspieler werden jeweils nur für eine bestimmte Rolle verpflichtet. Das finanzielle Risiko des Theaatersleiters wird in manchen Fällen dadurch reduziert, dass den Darstellern keine fixe Gage, sondern ein Anteil an den Einnahmen zugesichert wird.

Das Bundesministerium für Unterricht, die für die Förderung der Kunst zuständige staatliche Stelle unterstützt einige Kellerbühnen, die ihre künstlerische Leistungsfähigkeit durch längere Zeit nachgewiesen haben. Die Gemeinde Wien wendet bei der Förderung der Kleinbühnen. ein Prämiensystem an. Die kleinen Theater erhalten je nach den Grad der Anerkennung, welche die Aufführung eines Stükkes bei der Begutachtung durch eine Jury findet, Prämien in gestaffelter Höhe.

\section{"Theater der Jugend" - "Volkstheater in den Aussenbezirken"}

Es ist noch auf zwei seit Jahren erfolgreiche Einrichtungen im Wiener Theaterleben hinzuweisen, die ausserhalb eines traditionellen Theaterbetriebes darum bemüht sind, neues Publikum zu gewinnen, Theaterbesucher für heute und für morgen.

Das "Theater der Jugend", eine von der Schulbehörde subventionierte Vereinigung, produzierte in der vergangenen Spielzeit an drei Spielstätten in Wien sechzehn Stücke, Märchenspiele für die Volksschüler, Klassiker und moderne Problemstücke für die Schüler höherer Lehranstalten. Ausserdem wird durch diese Institution Kindern und Jugendlichen in ausschliesslich ihnen vorbehaltenen, verbilligten Vorstellungen der Besuch der grossen Wiener Bühnen und der Kellertheater ermöglicht, sofern die Darbietungen für Jugendliche geeignet und vom entsprechenden literarischen Wert sind. Abonnements-und Einzelkarten für das Theater der Jugend werden in den Schulen verkauft. 
In jeder Lehranstalt ist eine Lehrkraft damit betraut, dort die Agenden des Theaters der Jugend zu führen. In ähnlicher Weise arbeitet der österreichische Zweig der internationalen Vereiningung "Jeunesses musicales" durch ihn werden den Schülern der höheren Lehranstalten Abonnements für Konzertzyklen und Karten für Einzelveranstaltungen sehr preisgünstig angeboten.

Das "Theater der Jugend" gibt seit vielen Jahren eine sehr bemerkenswerte Kulturzeitschrift für Junge Menschen unter dem Titel "Neue Wege" heraus. In diesen Heften werden Aufsätze und Rezensionen publiziert, die auf den Theaterbesuch vorbereiten. Die Zeitschrift enthält aber auch literarische Beiträge junger Dichter und Schriftsteller. Es wäre unmöglich, die Entwicklung der neuen österreichischen Lyrik zu studieren, ohne sich mit den "neuen Wegen" zu beschäftigen.

In den letzten zwei Jahrzehnten wurden in Wien drei groBe, jederzeit bespielbare Theatergebäude abgerissen. Die Erregung darüber war gross. Wie konnte so etwas in der Theaterstadt Wien passieren? Der damals für die Kultur zuständige Stadtrat bereuerte, dass dieses Theater, wenn sie fahrbar gewesen wären, von der Demolierung bewahrt worden wären. Das heisst, dass man diese Bühnen an ihren Stadtorten in den unmittelbar an die City angrenzenden Stadtteilen für entbehrlich hielt, dass man sie aber in den am Stadtrand gelegenen Wohnvierteln benötigt hätte.

Nun, man hat in den Randbezirken zwar keine neuen Theater errichtet, wohl aber dort und da auch für Theateraufführungen geeignete Mehrzwecksäle gebaut. In diesen und in anderen. - älteren - Räumen - an insgesamt etwa zwanzig Spielstätten - gibt das Ensemble des Volkstheaters im Laufe eines Monats je eine Vorstellung eines für diese Tournée einstudierten Stückes. Die Kleinheit der Bühnen und der Mangel an komplizierteren technischen Einrichtungen schliessen die Aufführung personenreicher Stücke und solcher, die häufigen Szenenwechsel erfordern, aus.

Zweck dieser Tournéen durch die Aussenbezirke, die von der Arbeiterkammer, der Berufsvertretung der Arbeiter und Angestellten, finanziell unterstützt werden, ist die Gewinnung eines neuen Theaterpublikums, vor allem aus den Kreisen der Arbeiterschaft. Eine mehr als zehnjährige Erfahrung zeigt, dass auf diese Weise das Interesse an Theater tatsächlich geweckt oder belebt werden konnte. Die Frequenz der Vorstellungen ist aber gerade in den ausgesprochenen Arbeiter- 
bezirken rückläufig. Die Konkurrenz durch das Fernsehen spielt dabei sicher eine gewisse Rolle. Aber auch die weit fortgeschrittene Motorisierung der Arbeiter und kleinen Angestellten ist für den Publikumsrückgang mitverantwortlich. Der Besitz eines Autos erleichtert den Besuch der eleganteren Bühnen in der Innenstadt. Ein solcher wesentlich teuerer Theaterbesuch befriedigt aber auch den Wunsch, sein Sozialprestige $\mathrm{zu}$ steigern.

Sollte die Aktion "Das Volkstheater in den Aussenbezirken" ihren Zweck -die Gewinnung neuen Publikums- ganz erfüllen, müsste man die Jugend besonders anzusprechen trachten. Das hätte natürlich eine Aenderung des Spielplanes zur Voraussetzung. Dabei dürfe aber nicht von vornherein angenommen werden, dass jene avangardistischen Stücke, mit denen man das Interesse der Jugend in Zentrum anspricht, auch am Stadtrand erfolgreich wären. Zweifellos aber würde eine Programmgestaltung mit stärkerer Berücksichtigung der modernen Dramatik den Verlust von Teilen des Stammpublikums, das sich vor allem aus älteren Menschen rekrutiert, zur Folge haben.

\section{Theater in den Bundesländern}

Die Republik Österreich ist ein Bundesstaat, der von neun Bundesländern gebildet wird. Die Bundeshauptstadt Wien ist eines dieser Bundesländer. Von den übrigen acht besitzt eines, nämlich Niederösterreich, welches das Territorium von Wien allseitig umschliesst, in zwei Städten ständig bespielten Bühnen. An ihnen werden die Operette und das Sprechstück gepflegt. In fünf Bundesländern gibt es nur in deren Hauptsätdten ständige Theater, die alle Ensembles für das Schauspiel, die Oper und die Operette engagiert haben und auch über grössere oder kleinere Balettgruppen verfügen. Auch die restlichen zwei Bundesländer verfügen über Theater, die allerdings nur das Schauspiel pflegen und als Wanderbühnen geführt werden.

Graz, die zweitgrösste Stadt Österreichs, besitzt ein repräsentatives Opernhaus mit 1400 Plätzen und ein sehr stilvolles altes, durch Umbau den modernen Bedürfnissen und Sicherheitsvorschriften angepasstes Schauspielhaus.

In Linz, der rasch wachsenden Stadt an der Donau, wird in zwei Theatern, dem sogennannten Grossen Haus und in den Kammerspielen ständig gespielt. Beide Bühnen, wurden in ihrer jetzigen Gestalt nach dem Zweiten Weltkrieg geschaffen. 
Das Tiroler Landestheater in Innsbruck verfügt seit kurzem über.ein neues Grosses Haus, dem jedoch die künstlerisch bedeutende klassizistische Fassade des alten Theaters erhalten blieb. In umittelbarer Nähe dieses Landestheaters befinden sich die geräumigen Kammerspiele. Experimentelle Stücke werden in Innsbruck auf einer Probebühne dargeboten.

Die Städte Salzburg und Klagenfurt begnügen sich derzeit mit Vorstellungen in einem Haus. Das Klagenfurter Theatergebäude aus dem Jahre 1908 wurde vor einigen Jahren durch einen Zubau erweitert. In Salzburg steht ein Umbau bevor.

Das Theater für Vorarlberg verfügt in der Hauptstadt Bregenz über ein durch Umgestaltung eines historischen Gebäudes entstandenes modernes Theater, das auch von den Bregenzer Festspielen als Spielstätte benützt wird. Die Bühne in Bregenz wird freilich nicht durchlaufend bespielt, denn das Theater für Vorarlberg, also für das kleine Land im äussersten Westen Österreichs, wird ebenso wie die burgenländische Landesbühne - im äussersten Osten Österreichs - als Wanderbühne geführt. Dadurch wird in einer verhältnismässig grossen Anzahl von Orten die Möglichkeit geboten, einigermassen regelmässig an Theatervorstellungen teilnehmen zu können. Diesem Ziel dienen aber auch die schon erwähnten standortgebundenen Bühnen in den österreichischen Bundesländern durch sogenante "Abstecher". Das sind Vorstellungen, die ausserhalb des Standortes stattfinden. So spielten zum Beispiel die Ensembles der Grazer Theater in der vergangenen Saison in 13, das Innsbrucker Theater in 12 Orten. Mehrere Bühnen veranstalten aber auch in ihrem Stammhaus regelmässig Abonnementvorstellungen für Besucher von auswärts. Das Publikum wird sofern es nicht eigene Autos benützt, mit Autobussen zu den Vorstellungen gebracht.

Noch vor 35 oder 40 Jahren haftete den österreichischen Theatern ausserhalb von Wien und Graz das Odium des Provinziellen, oder gar der Schmiere an. Es wurden damals erbärmlich niedrige Gagen bezahlt, die Ausstatung der Stücke erfolgte aus einem kleinen Fundus von Dekorationen und Kostümen, die jedem Stammgast längst bis zum Überdruss bekannt waren. Die Ensembles waren klein und die Stücke -nur Operetten bildeten wohl eine Ausnahme- brachten es zu wenigen Aufführungen. Eine Premiere jagte die andere, die Probezeit war daher ganz kurz, die Schauspieler waren überanstrengt, das Künstlerische Ergebnis daher oftmals unbefriedigend. 
Dass sich die Situation an den sogenannten Provinzbühnen wesentlich gebessert hat, dass die meisten Aufführungen durchhaus erfreuliches künstlerisches Niveau erreichten und dass an manche auch die an wesentlich grösseren Bühnen üblichen Masstäbe angelegt werden können, wurde durch verschiedene Umstände ermöglicht. Einer ist in der Vergrösserung der Ensembles zu sehen. Dadurch ist es leichter geworden, die Stücke richtig zu besetzen. Dazu kommt, dass sich das Bildungsniveau der Schauspieler im Allgemeinen beträchtlich gehoben hat. Ihrer Ausbildung dienen, -das sei hier nebenbei erwähnt- Schauspielseminare an den drei staatlichen Akademien für Musik und Darstellende Kunst in Wien, Salzburg (Mozarteum) und Graz. Die staatliche Wiener Schauspielschule wurde in ihrer jetzigen Form von Max Reinhardt, dem grossen Regisseur der Zwischenkriegszeit, gegründet. Sie trägt auch heute noch den Namen Max Reinhardt-Seminar. Neben den staatlichen gibt es auch private Schauspielschulen, die guten Ruf geniessen.

Doch zurück zu den Bühnen in den österreichischen Bundesländern! Die Theaterorchester sind heute - verglichen mit der Situation vor etwa 40 Jahren - reicher und mit besser ausgebildeten Kräften besetzt. Auch von den Ballettruppen lässt sich Aehnliches behaupten, wenn auch den Tanzvorführungen an den österreichischen Provinzbühnen keine dominierende Bedeutung zukommt.

Es war schon von den einstmals ärmlichen Fundus dieser Provinztheater die Rede. Heute wäre es an allen diesen Bühnen undenkbar, ein Stück einfach aus den Fundus auszustatten. Es wird selbtverständlich für jede Inszenierung eine eigene Dekoration neu hergestellt. An allen Theatern sind Bühnen und Kostümbildner fest verplichtet. Ihrer Ausbildung dienen in Österreich zwei Meisterklassen und zwar an der Akademie der Bildenden Künste in Wien und an der Akademie für Angewandte Kunst ebenfalls in Wien.

Auch die technische Einrichtungen an den Theatern wurden in den letzten Jahrzehnten modernisiert und erweitert. Selbstverständlich haben auch diese Investitionen zur Verbesserung des künstlerischen Niveaus der Vorstellungen ihren Teil beigetragen.

Voraussetzung für diese Aufwärťsentwicklung war selbstverständlich eine bessere Dotierung der Bühnen. Die Stadtverwaltungen und die Landesregierungen, die in der Regel einen gemeinsamen Theaterausschuss bilden, fungieren gemeinsam als Theatererhalter. Diese Erhalter der österreichischer Theater - mit Ausnahme der Bundestheater 
und der Wiener Privattheater- haben sich zu dem "Theatererhalterverband der österreichischer Bundesländer - und Städte" zusammengeschlossen. Dadurch soll eine einheitliche Haltung der Bühnen in sozialrechtlichen Fragen und bei Verhandlungen mit der Gewerkschaft gewährleistet werden. Auch die Anteile, die den einzelnen Theatern aus einer staatlichen Globalsubvention zukommen, wurden von Theatererhalterverband festgelegt. Eine Forderung des Verbandes zielt darauf die staatliche Förderung der Provinzbühnen in eine fixe Relation zu den ständig steigenden Summen zu setzen, die der Staat für die von ihm erhaltenen Theater, die Bundestheater, ausgibt.

Als Beispiel für den Finanzbedarf eines solchen österreichischen Provinztheaters möchte ich die Gebarung des Salzburger Landestheaters also einer der kleineren Bühnen in den Bundesländern, anführen. In der vergangenen Spielzeit betrugen die Ausgaben 22,6 Millionen Schilling, das sind, 11,5 Min T lira, die Einnahmen 6,7 Millionen Schilling, das sind 3,4 000 Tl Auf Grund eines solchen Verhaltnisses der Ausgaben zu den Einnahmen - diese konnten hier fast ein Drittel der Ausgaben decken - kann das Salzburger Theater auf die perzentuell günstigsten Einspielergebnisse von allen österreiChischen Bühnen in den Bundesländern hinweissen.

\section{Festspiele}

Mit einigen kurzen Worten seien abschliessend noch die zahlreichen Festspielveranstaltungen in Österreich erwähnt. Die Salzburger Festspiele sind in den fünfzig Jahren Bestehens zu einer international so bekannten Einrichtung geworden, dass ich es mir versagen kann, über ihre Darbietungen, aber auch über ihre Bedeutung für das Kulturprestige Österreichs und für den Fremdenverkehr des Landes zu sprechen. Vielleicht aber soll von der negativen Seite dieser glanzvollen Veranstaltungen, nämlich vom Defizit die Rede sein und von der Regelung die getroffen wurde, um es alljährlich abzudecken. Vor zwanzig Jahren wurde ein Gesetz geschaffen, durch das der Staat verpflichtet wird, $40 \%$ dieses Defizits zu tragen. Für je zwanzig Prozent haben alljährlich das Land Salzburg, die Stadt Salzburg und der Salzburger Fremdenverkehrsfond aufzukommen. Herbert von Karajan hat vor einigen Jahren seiner Geburtsstadt Salzburg mit den Osterspielen eine kurze 2 Festspielsaison geschenkt, deren. Programm bisher Opern Richard Wagners und Konzerte umfasste. Der grosse Dirigent hat das Projekt zunächst dusch Einsatz eigener Mittel veranstaltet. Der Fortbestand wurde durch eine Vereinigung von Abonnenten 
und durch relativ geringe Zuschüsse des Landes Salzburg gesichert Um eine gesetzliche Regelung, welche die Deckung des Defizits Wie für Salzbung sichert, bemühen sich seit Jahren die Bregenzer Festspiele und die Wiener Festwochen.

Die Hauptattraktion der Bregenzer Festspiele sind Operetten-oder Ballettaufführungen auf einer im Bodensee installierten Spielfläche. Diese Seespiele üben grosse Anziehungskraft auf das Publikum aus dem benachbarten Teilen der Deutschen Bundesrepublik und aus der Schweiz aus. Die Bregenzer Festspiele bieten alljährlich auch dem Burgteater die Möglichkeit, seine Kunst im Westen Österreichs zu zeigen.

In das sehr reichhaltige Programm der Wiener Festwochen sind neben den schon erwähnten Eigenproduktionen der Festwochenintendanz glanzvolle Aufführungen aller Wiener Theater, zahlreiche Gast. spiele ausländischer Ensembles einbezogen. Überaus reichhaltig ist das Konzertprogramm der Wiener Festwochen.

Konzerte und Musiktheater dominieren auch im Programm des "Steierischen Herbstes", der mit festlichen Aufführungen in Graz begangen wird . Während die übrigen Festspiele dem Publikumsgeschmack durch eine vorwiegend konservative Programmbildung entgegenkommen, widmen sich die Wiener Festwochen und noch viel kompromissloser der "Steierische Herbst" der Moderne. Die Grazer Veranstaltungen pflegen bei der Programmbildung und bei der Auswahl der Mitwirkenden sehr bewusst die kulturellen Kontakte zu den südlichen Nachbarländern Österreichs, nämlich zu Jugoslawien und Italien.

Von dem Bregenzer Beispiel inspiriert, verantaltet man auch auf dem Neusiedlersee, im äussersten Osten Österreichs, Seespiele. Diese Operettenaufführungen sind vor allem für das Wiener Publikum bequem zu erreichen. Noch näher von der österreichischen Hauptstadt liegt die Burg Forchtenstein, in deren Burggraben alljährlich ein Werk des grössten österreichischen Dramatikers Franz Grillparzer inszeniert wird.

Im Süden Österreichs werden im Hof des herrlichen Renaissanceschlosses der kleinen Stadt Spittal ebenfalls im Hochsommer sehr ambitioniert und beschwingt Komödien gespielt. In dem nicht weit von Spittal entfernten Friesach spielen Bewohner dieser kleinen Stadt -also Laienspieler- nun schon seit vielen Jahren erfolgreich Theater. 
Ihr Programm bevorzugt grosse Dramen der Weltliteratur. In einigen Tiroler Dörfern werden von den Ortsbewohnern spiele von Leiden und Sterben Jesu Christi, die sogenannten Passionsspiele dargestellt.

Mit diesen kurzen Hinweisen auf die sommerlichen Festspiele in Österreich strebe ich keine Vollständigkeit an. Es wird in dieser Jahreszeit ja mancherorts eine Bühne für ein Spiel unter freiem Himmel aufgeschlagen. Und wenn solche Sommerveranstaltungen auch besonders auf die Bedürfnisse des Fremdenverkehrs Bedacht nehmen, so geht es dabei doch nicht nur um das Geschäft. In diesen Spielen manifestiert sich -wie einstmals in den glanzvollen Auführungen der Barockzeit-auch heute noch das Theater als F e st, das die Österreicher ihren Gästen aus aller Welt und sich selbst bereiten. 


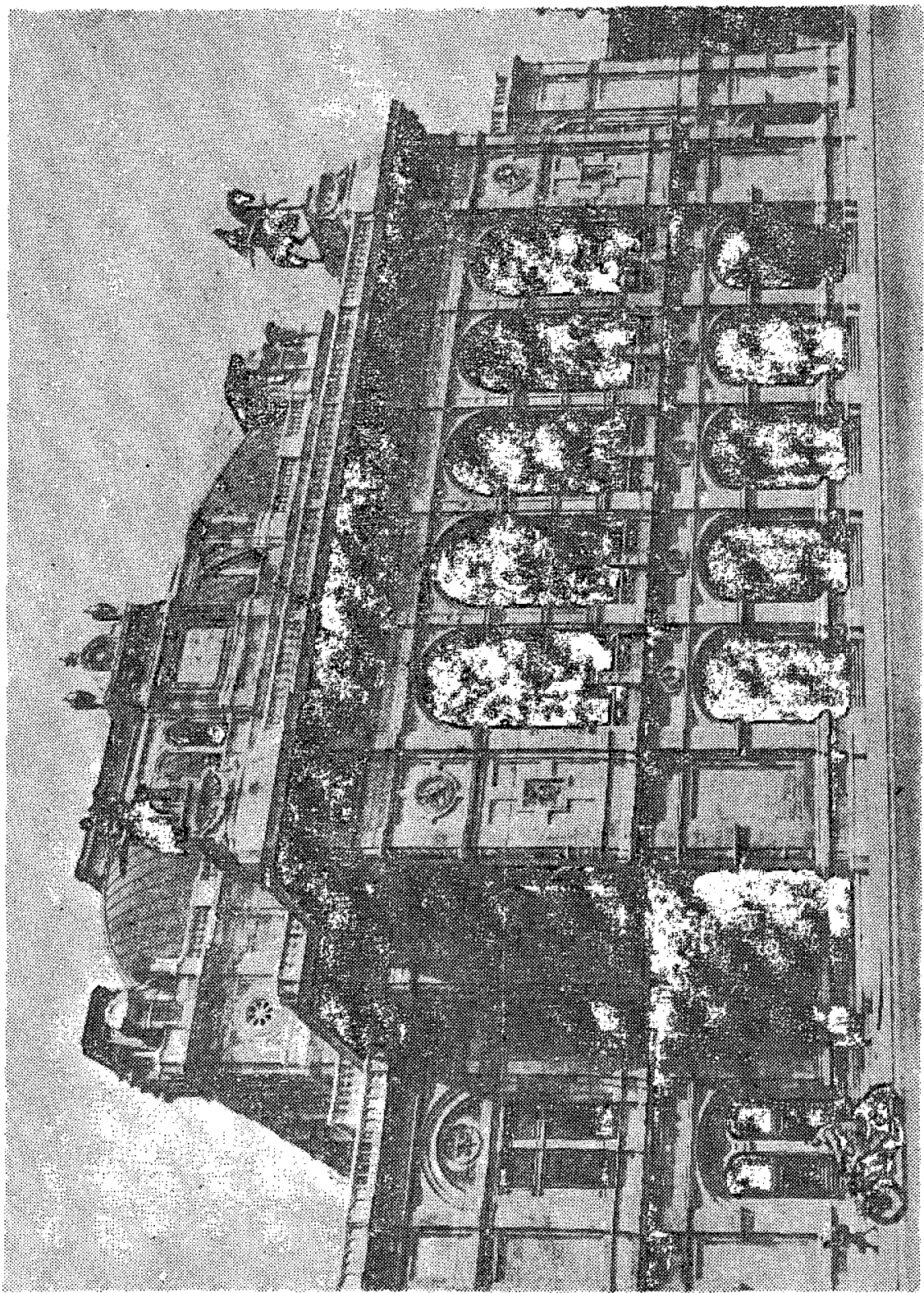




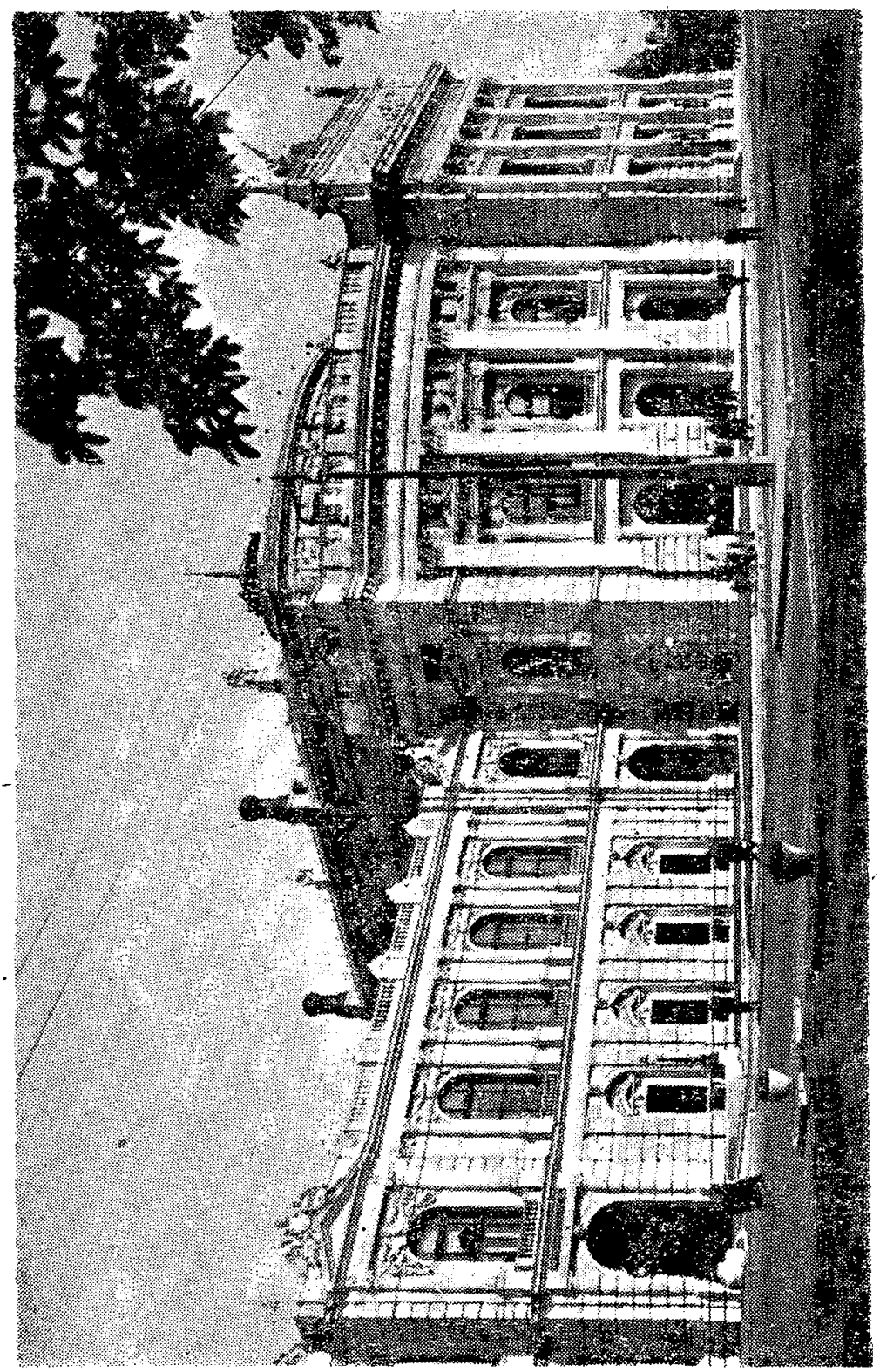

